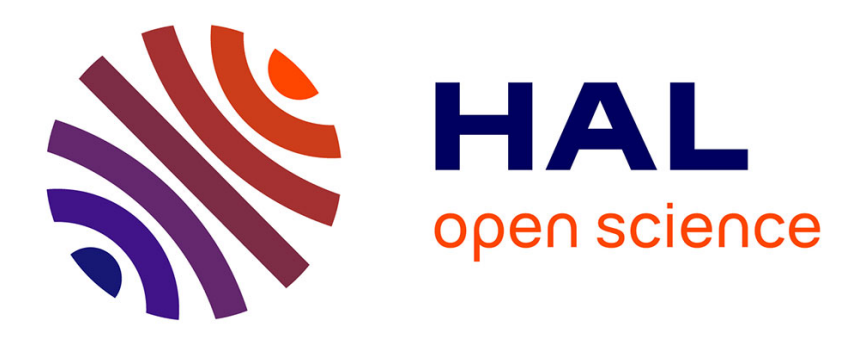

\title{
Modelling heavy doping effects for low temperature device simulations
}

\author{
S. Sokolic, S. Amon
}

\section{To cite this version:}

S. Sokolic, S. Amon. Modelling heavy doping effects for low temperature device simulations. Journal de Physique IV Proceedings, 1994, 04 (C6), pp.C6-133-C6-138. 10.1051/jp4:1994621 . jpa-00253115

\section{HAL Id: jpa-00253115 https://hal.science/jpa-00253115}

Submitted on 1 Jan 1994

HAL is a multi-disciplinary open access archive for the deposit and dissemination of scientific research documents, whether they are published or not. The documents may come from teaching and research institutions in France or abroad, or from public or private research centers.
L'archive ouverte pluridisciplinaire HAL, est destinée au dépôt et à la diffusion de documents scientifiques de niveau recherche, publiés ou non, émanant des établissements d'enseignement et de recherche français ou étrangers, des laboratoires publics ou privés. 


\title{
Modelling heavy doping effects for low temperature device simulations
}

\author{
S. Sokolić and S. Amon
}

Faculty of Electrical and Computer Engineering, University of Ljubljana, Tržaška 25, 61000 Ljubljana, Slovenia

\begin{abstract}
A new temperature dependent apparent bandgap narrowing model, appropriate for low temperature device simulations is proposed. The model is based on the decomposition of apparent bandgap narrowing in equilibrium into different contributions at $300 \mathrm{~K}$ and on the theoretical temperature dependence of each phenomenon. Specific heavy doping effects that can be important at low temperatures are discussed. Temperature dependence of apparent bandgap narrowing in equilibrium is more significant at high concentration of ionized impurities, where apparent bandgap narrowing decreases with decreasing temperature, as a consequence of temperature dependent degeneracy.
\end{abstract}

\section{INTRODUCTION}

Heavily doped regions exist in almost every silicon device operating at low temperatures. Increased interest in low-temperature operation of semiconductor devices requires for improved simulation tools, appropriate for low-temperature device modelling. For the purposes of device modelling, heavy doping effects are almost universally described with apparent bandgap narrowing in equilibrium $\Delta G_{0}$ or closely related effective intrinsic concentration in equilibrium $n_{\text {ieffo. }}$. The relatively straightforward empirical validation of $\Delta G_{0}$ and its simplicity are the most important reasons for the popularity of this approach. As shown in [1,2], $\Delta G_{0}$ consists of two main contributions: - actual bandgap narrowing in equilibrium $\Delta E_{g 0}$ as a consequence of actual band distortion influencing steady-state transport of minority carriers (shift of band edges, formation of band tails and other distortion mechanisms), and - degeneracy in equilibrium $\Delta E_{F D O}$ as a result of the invalidity of Boltzmann statistics in the high doping regime:

$$
p_{0} n_{0}=n_{\text {ieffo }}^{2}=n_{i 0}^{2} \exp \left(\frac{\Delta G_{0}}{k T}\right)=n_{i 0}^{2} \exp \left(\frac{\Delta E_{g 0}+\Delta E_{F D O}}{k T}\right) .
$$

During the past two decades, a lot of experimental and theoretical work studying doping dependence of $\Delta G_{0}$ at room temperature has been done $[3,4,5]$. The authors in [3] illuminated the theory underlying the electrical characterization of heavily doped regions and the assumptions necessary for extraction of $\Delta G_{0}$. In contrast with room temperature work, little theoretical and experimental work concerning $\Delta G_{0}$ at low temperatures has been performed. However, two approaches related to the modelling of $\Delta G_{0}$ at low temperatures are most frequently in use: - the apparent bandgap narrowing is considered as temperature independent, and - pure theoretical models with their temperature dependence are employed. The purpose of this work is to present a new temperature dependent apparent bandgap narrowing model, appropriate for device simulations. The derivation is based on critical analysis of different phenomena involved in $\Delta G_{o}$ at room temperature, and the theoretical prediction of the Article published online by EDP Sciences and available at http://dx.doi.org/10.1051/jp4:1994621 
temperature dependence of each phenomenon. We found that apparent bandgap narrowing is a strong function of temperature, especially in $p$-type silicon at high concentration of impurities.

\section{APPARENT BANDGAP NARROWING AT 300K}

For the purposes of analysis, a heavily doped $N^{+}$region in quasi-equilibrium was considered. The validity of Boltzmann statistics for minority carriers was assumed. By means of the rigid band model [1], the equilibrium carrier concentrations can be described with (2) and (3):

$$
\begin{gathered}
n_{0}=N_{C} \frac{2}{\sqrt{\pi}} \mathrm{F}_{1 / 2}\left(\frac{E_{F}-E_{C O}}{k T}\right), \\
p_{0}=N_{v} \exp \left(\frac{E_{V 0}-E_{F}}{k T}\right),
\end{gathered}
$$

where $N_{C}$ and $N_{V}$ are effective densities of states in conduction and the valence band, respectively; $\mathrm{F}_{1 / 2}$ is Fermi-Dirac integral of order $1 / 2$, and $E_{C O}$ and $E_{V 0}$ are conduction and valence band edges, respectively. The actual bandgap narrowing is determined by the position of the band edges, and degeneracy is included in the Fermi-Dirac integral. Taking into account (1), (2), (3), together with the space charge neutrality assumption [6], the following relationship can be obtained:

$$
N_{D}^{*}=N_{C} \frac{2}{\sqrt{\pi}} F_{1 / 2}\left(\frac{E_{v 0}-E_{C O}}{k T}-\ln \left(\frac{n_{i 0}^{2} \exp \left(\frac{\Delta G_{0}}{k T}\right)}{N_{V} N_{D}^{+}}\right)\right) \text {. }
$$

If we further consider the relation between intrinsic carrier concentration in equilibrium $n_{i 0}$ and bandgap in the absence of heavy doping effects $E_{g 0}{ }^{\circ}\left(n_{i 0}{ }^{2}=N_{C} N_{V} \exp \left(-E_{g 0}{ }^{\circ} / k T\right)\right)$, and take into account the inverse Fermi function of order $1 / 2\left(\mathrm{G}_{1 / 2}\left(2 \pi^{-1 / 2} \mathrm{~F}_{1 / 2}(x)=x\right)\right.$, the following expressions for $\Delta E_{g 0}(5)$ and $\Delta E_{F D O}(6)$ can be derived:

$$
\begin{gathered}
\Delta E_{g 0}\left(N_{D}^{*}, T\right)=\Delta G_{0}\left(N_{D}^{*}, T\right)+k T \mathrm{G}_{1 / 2}\left(\frac{N_{D}^{*}}{N_{C}(T)}\right)-k T \ln \left(\frac{N_{D}^{*}}{N_{C}(T)}\right), \\
\Delta E_{F D 0}\left(N_{D}^{+}, T\right)=-k T \mathrm{G}_{1 / 2}\left(\frac{N_{D}^{+}}{N_{C}(T)}\right)+k T \ln \left(\frac{N_{D}^{+}}{N_{C}(T)}\right) .
\end{gathered}
$$

Analogous expressions can be derived for heavily doped $P^{+}$regions as well. If the concentration of ionized impurities is known - at room temperature total ionization of impurities can be assumed $-\Delta E_{g 0}$ and $\Delta E_{F D 0}$ can be recalculated from any $\Delta G_{0}$ data.

Our analysis was based on the experimental $\Delta G_{0}$ data given in [4] (data from many authors recalculated for the unified mobility model) and [5]. The values for $N_{C}, N_{V}$ and $E_{g 0}{ }^{0}$ were taken from [7]. $G_{1 / 2}$ was approximated according to [8]. Since most of the experimental procedures need the value of $n_{i 0}$ to render $\Delta G_{0}$ [4], the experimental data were first recalculated to be consistent with our set of $N_{C}, N_{v}$ and $E_{g 0}{ }^{0}$ according to the procedure described in [4]. $\Delta E_{g 0}$ data obtained from recalculated $\Delta G_{0}$ data are presented in Fig. 1. Good agreement between $p$ - and $n$-type data is retained due to similar degeneracies in $p$ - type and $n$ - type silicon at room temperatures.

The shift of band edges was assumed to be the dominant mechanism present in the actual bandgap 
narrowing. Therefore, the theoretical model for band edge shifts was used in order to find a fit for $\Delta E_{g o}$ data. Although different dependencies for gap shift in $p$ - and $n$-type of silicon are proposed by theoretical models, we neglected this difference because of good agreement between existing $n$ - and $p$-type experimental data, and because of the small quantity of $p$-type data at doping concentrations higher then $10^{19} \mathrm{~cm}^{-3}$. Complete $\Delta E_{g 0}$ data were in best agreement with the model presented in [9] describing the doping dependence of $\Delta E_{g 0}$ in $n$-type silicon. The authors in [9] found $N^{1 / 2}$ dependence at low doping concentrations and $N^{1 / 4}$ dependence at high doping concentrations, with transition point around $210^{18} \mathrm{~cm}^{-3}$. $\Delta E_{g o}$ data were therefore fitted with (7)

$$
\Delta E_{g 0}=\left(\left(a_{300} N^{1 / 2}\right)^{-c}+\left(b_{300} N^{1 / 4}\right)^{-c}\right)^{-1 / c}
$$

where $N$ is ionized donor or acceptor concentration $\left[\mathrm{cm}^{-3}\right]$, and $a_{300}, b_{300}$ and $c$ are fitting parameters $(c$ determines the sharpness of transition between two dependencies). The best fit obtained with $a_{300}=4.373$ $10^{-11}\left[\mathrm{eV} \mathrm{cm}{ }^{3 / 2}\right], b_{300}=1.27210^{-6}\left[\mathrm{eV} \mathrm{cm}^{3 / 4}\right]$ and $c=4$ is presented in Fig.1. Optical measurements of gap narrowing [2] were found to be in good agreement both with electrically obtained experimental data and with the theory (Fig.1), confirming the assumption of rigid shrinkage as a dominant actual bandgap narrowing mechanism.

Finally, by combining theoretical expressions for degeneracy (6) and experiment-based fit for actual bandgap narrowing (7), the apparent bandgap narrowing is obtained. Detailed discussion concerning analysis of apparent bandgap narrowing at $300 \mathrm{~K}$ and comparison with other approaches and models is given in [10].

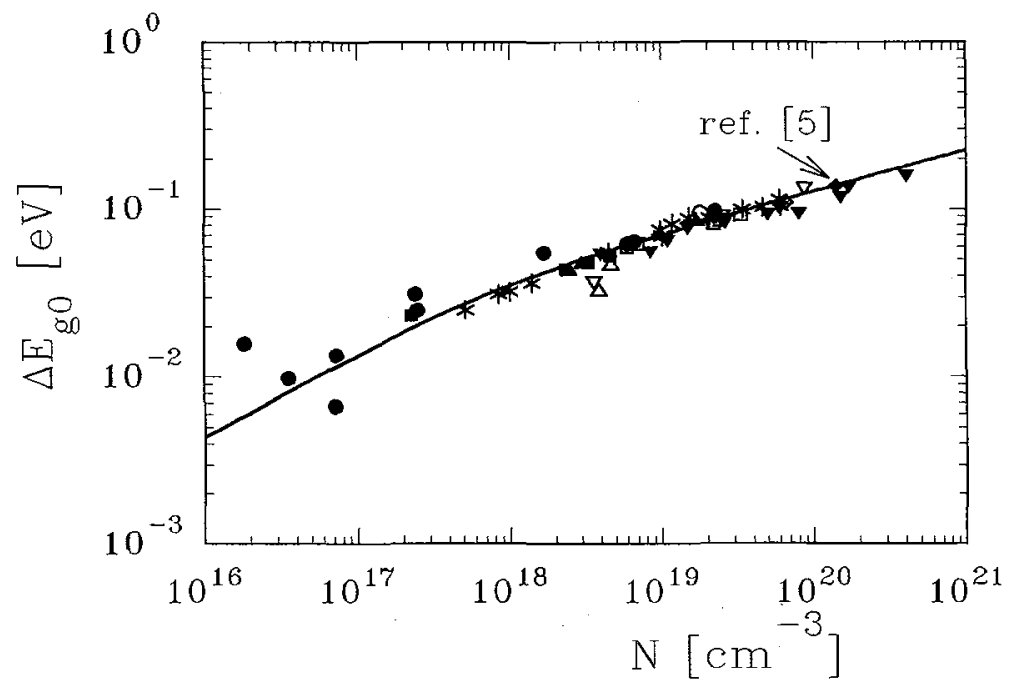

Fig.1: Doping dependence of actual bandgap narrowing. All symbols except $\boldsymbol{\nabla}$ correspond to the actual bandgap narrowing obtained from experimental apparent bandgap narrowing data: $\bullet$ is taken from [5], all others are taken from [4] (notation is the same as in [4]). All data except $\bullet$ and $\Delta$ were recalculated for this work $n_{i 0}$. $\nabla$ represents optical data taken from [2]. The solid line represents the best fit for actual bandgap narrowing based on electrical data (eq. (7)).

\section{TEMPERATURE DEPENDENCIES OF $\Delta E_{g 0}, \Delta E_{F D O}$ and $\Delta G_{0}$}

\subsection{Temperature dependence of $\Delta E_{g}$}

To find the temperature dependence of $\Delta E_{g 0}$, the theoretically predicted temperature dependence of rigid gap shrinkage was considered. As suggested in [9], the $n^{I / 4}$ part of the $\Delta E_{g 0}$ curve describing 
actual bandgap narrowing in the high doping concentration regime is temperature independent. By contrast, the low concentration portion of $\Delta E_{g 0}$ curve is temperature dependent; according to [9] it exhibits $T^{1 / 2}$ temperature dependence. These theoretical results are in reasonable agreement with temperature dependence of optical data [2], reported for $20 \mathrm{~K}$ and recalculated for $300 \mathrm{~K}$. Therefore, to obtain the temperature dependent expression for $\Delta E_{g 0}, a_{300}$ term in (7) is replaced with $a(T)\left(a(T)=a_{300}(T / 300)^{-t / 2}\right)$. The results are presented in Fig. 2 .

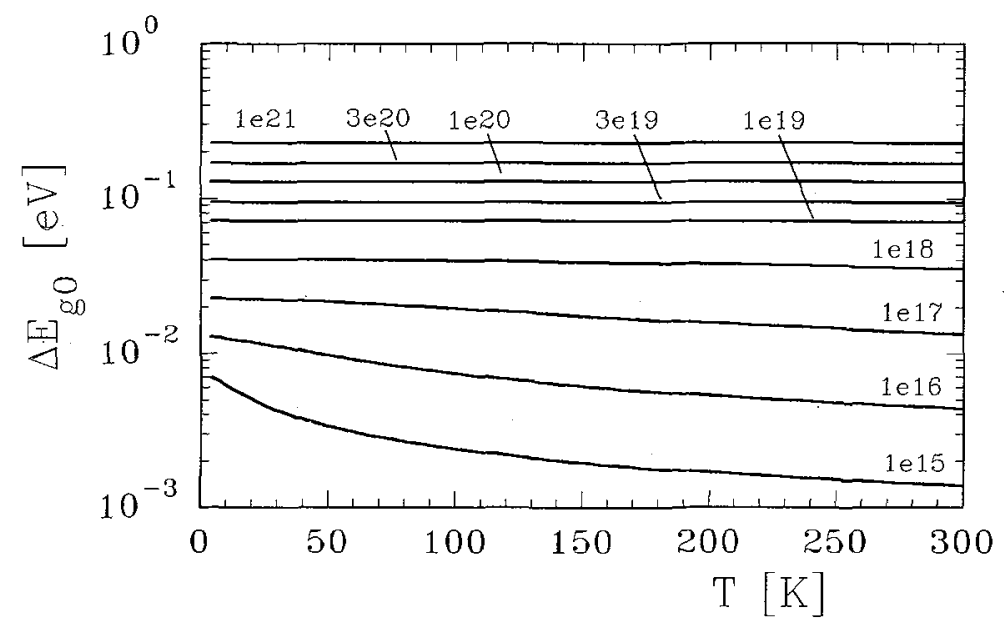

Fig.2: Temperature and ionized doping dependence of actual bandgap narrowing, calculated according to (7).

\subsection{Temperature dependence of $\Delta E_{F D O}$}

The temperature dependence of conduction and valence band degeneracy was calculated according to (6) and its counterpart for $p$-type silicon. Temperature dependencies of $N_{C}$ and $N_{V}$ were taken from [7]. The results are presented in Fig.3. It is obvious that degeneracy is much more temperature dependent than actual bandgap narrowing, especially in $p$-type silicon.

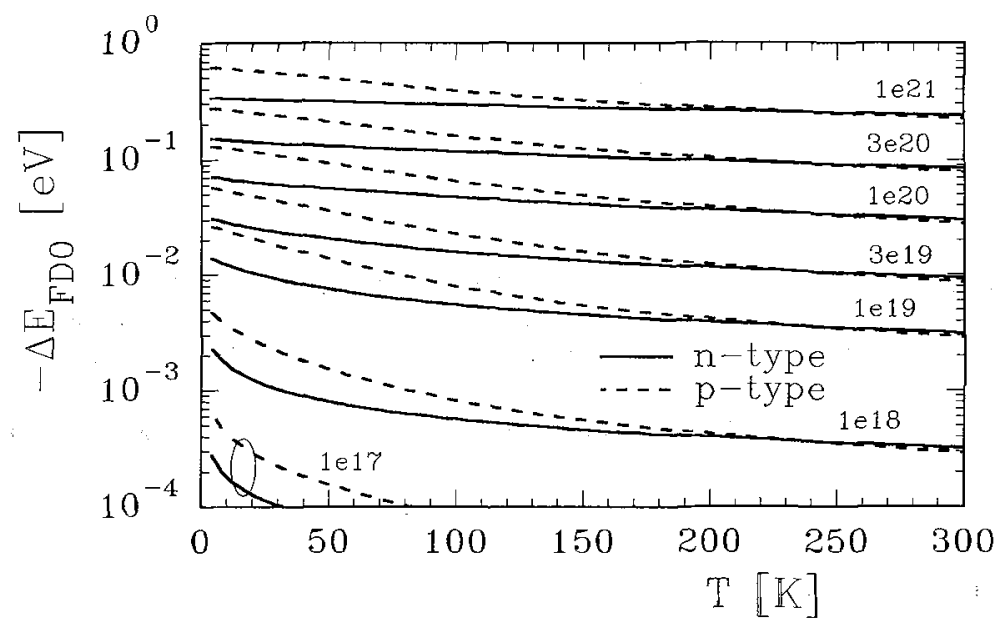

Fig.3: Temperature and ionized doping dependence of degeneracy, obtained from (6). 


\subsection{Temperature dependence of $\Delta G_{0}$}

The temperature dependence of apparent bandgap narrowing was obtained as a sum of temperature dependent actual bandgap narrowing and temperature dependent degeneracy. The results are presented in Fig.4. The temperature dependence of $\Delta G_{0}$ in the low ionized impurity concentration regime is a consequence of the temperature dependency of $\Delta E_{g 0}$. This temperature dependence is not significant and can be neglected in comparison with temperature dependence at high concentration of ionized impurities $\left(>10^{18} \mathrm{~cm}^{-3}\right)$, which is a consequence of temperature dependent degeneracy. The temperature dependence is more pronounced in $p$-type silicon.

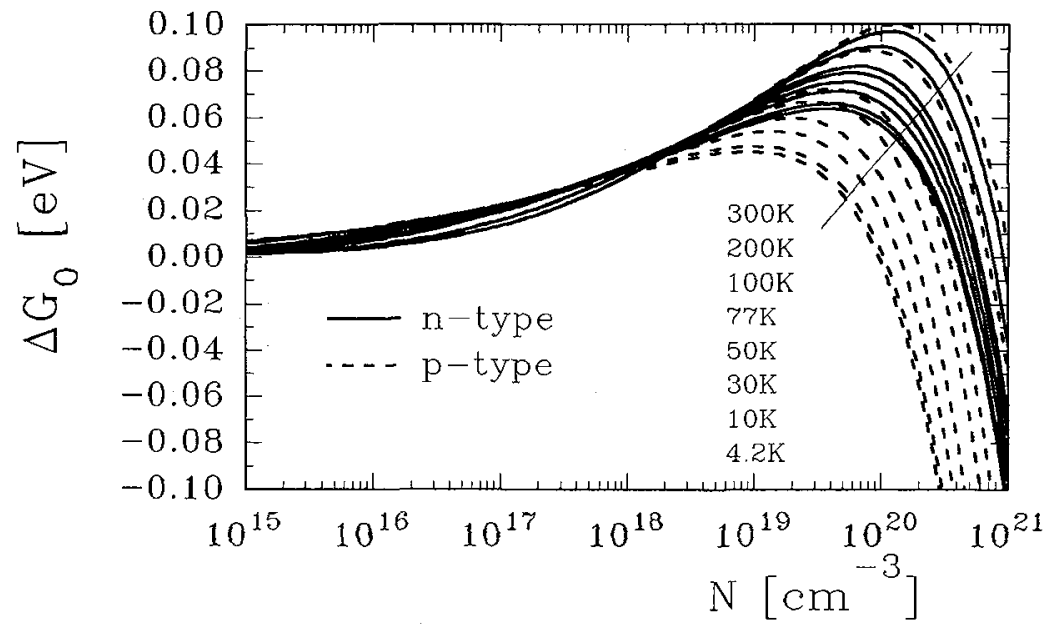

Fig.4: Temperature and doping dependence of apparent bandgap narrowing obtained by combining temperature and doping dependence of actual bandgap narrowing and degeneracy.

\section{DISCUSSION}

In order to verify the results obtained, it is necessary to compare our model with existing experimental data and with models used by other authors. Some apparent bandgap narrowing data measured at different temperatures are available in the limited temperature and doping range $(T>150 K$, $\left.N=210^{19} \mathrm{~cm}^{-3}, 1.410^{20} \mathrm{~cm}^{-3}\right)[4,5]$, and indicate the temperature independence of apparent bandgap narrowing. Our results are in reasonable agreement with this conclusion for a given temperature range. Comparison with other models is not straightforward, due to the lack of the work on modelling low temperature apparent bandgap narrowing, and due to frequent misuse of apparent bandgap narrowing in carrier concentration and transport equations (it is often neglected that degeneracy is embedded in $\Delta G_{0}$ ).

It is worth mentioning that our temperature dependent model for $\Delta G_{0}$ is an extension of the room temperature model to low temperatures. In other words, the definite validity of the model is restricted to the conditions valid in heavily doped emitters under low-level injection. In this case, use of ionized impurity concentration as an independent variable is straightforward. The model cannot be used in nonequilibrium carrier concentration and transport equation without generalization of actual bandgap narrowing - it is impossible to extract an arbitrary nonequilibrium value of actual bandgap narrowing from an equilibrium $p n$ product. Although appropriate for most practical conditions, this generalization is not valid in the high-level injection regime. Concentration of minority carriers, comparable to the concentration of majority carriers, is in disagreement with the assumptions employed through the derivation of our model and the assumptions employed through the derivation of the theoretical model for rigid band shrinkage [9]. The problem of high-level injection and related heavy doping effects at low temperatures has been discussed in [11]. In order to account for different apparent bandgap narrowing, resulting from high-level injection of minority carriers from the emitter to the base of an advanced bipolar transistor operating at low temperatures, the authors in [11] introduced the injection-induced bandgap 
narrowing.

Another uncertainty concerning extension of the room temperature apparent bandgap narrowing to low temperatures is related to minority carriers in the band tails, which are unimportant at room temperature. As apparent bandgap narrowing in equilibrium is a consequence of minority carrier transport [3], it can account for mobile minority carriers only. Since minority carriers in the tails are considered to be immobile, and their concentration is low compared to the concentration of mobile minority carriers at room temperature [12], they do not contribute to the apparent bandgap narrowing. As shown in [12], the portion of minority carriers in the tails in relation to those in the delocalized states increases rapidly with decreasing temperature. Although our low temperature apparent bandgap narrowing does not account for minority carriers in the tails, it is not yet clearly understood what the influence is of the high portion of minority carriers in the tails on minority carrier transport (apparent bandgap narrowing is primarily the minority carrier transport parameter).

Recent work [13] emphasizes the importance of non-parabolicity in the $p$-type of silicon, modelled by doping dependence of effective mass. Non-parabolicity of the valence band negates the use of the rigid band model [1], complicating the inclusion of heavy doping effects in device modelling. Nevertheless, if the doping and temperature dependence of effective densities of states is known, the derivation of the apparent bandgap narrowing model can also be done for the non-rigid band model.

The uncertainties described emphasize the necessity for more theoretical and experimental work related to heavy doping effects at low temperatures. Moreover, they prove the adequacy of the proposed approach to the low temperature apparent bandgap narrowing, splitting the apparent bandgap narrowing into contributions of different physical origin.

\section{CONCLUSIONS}

A new approach to the temperature dependence of apparent bandgap narrowing in equilibrium is presented, based on the decomposition of $\Delta G_{0}$ into different contributions at $300 \mathrm{~K}$ and on the theoretical temperature dependence of each phenomenon. $\Delta G_{o}$ exhibits strong temperature dependence in the high doping regime, as a consequence of temperature dependent degeneracy. Due to the lack of adequate experimental data, verification of the model in the wide range of temperatures and doping concentrations is not yet possible. Although it is clear that the model presented cannot account for every heavy doping phenomenon that can be important at low temperatures, the authors believe that an improved model for heavy doping phenomena at low temperatures is proposed, which is appropriate for low temperature device simulations.

\section{REFERENCES}

[1] Marshak A.H., Shibb M.A., Fossum J.G. and Lindholm F.A., IEEE Trans. Electron Devices ED28 (1981) 293-298.

[2] Wagner J. and del Alamo J.A., J. Appl. Phys. 63 (1988) 425-429.

[3] del Alamo J., Swirhun S. and Swanson R.M., Solid-State Electron. 28 (1985) 47-54, and the references therein.

[4] Klaassen D.B.M., Slotboom J.W. and de Graaff H.C., Solid-State Electron. 35 (1992) 125-129, and the references therein.

[5] Popp J. and Weng J., Solid-State Electron. 35 (1992) 999-1003.

[6] del Alamo J.A., Appl. Phys. Lett. 39 (1981) 435-436.

[7] Green M.A., J. Appl. Phys. 67 (1990) 2944-2954.

[8] Nilsson N.G., Appl. Phys. Lett. 33 (1978) 653-654; Phys. Stat. Sol. (a) 19 (1973) K75-K79.

[9] Thuselt F. and Rösler M., Phys. Stat. Sol. (b) 130 (1985) 661-673.

[10] Sokolic S. and Amon S., submitted for publication.

[11] Cressler J.D., Tang D.D. and Yang E.S., IEEE Trans. Electron Devices ED-28 (1989) 2576-2586.

[12] Pan Y., Jain S.C., Kleffstra M. and Balk P., Solid-State Electron. 35 (1992) 791-796.

[13] Poortmans J., Jain S.C., Totterdell D.H.J., Caymax M., Nijs J.F., Mertens R.P.and Van Overstraeten R., Solid-State Electron. 36 (1993) 1763-1771. 